\title{
Developing a Minimally Invasive Cell-Based Model to Predict Response to Major Trauma
}

Kayla Gates ${ }^{1,3}$, Rebecca Nunge ${ }^{1,3}$, Jamila Adom ${ }^{2,3}$, Mark Kaplan²,3, Todd McKinley ${ }^{1,3}$

${ }^{1}$ Department of Orthopaedic Surgery

${ }^{2}$ Department of Microbiology and Immunology

${ }^{3}$ Indiana University School of Medicine

Background. Physical trauma results in a systemic inflammatory response. Preliminary research in orthopedic trauma patients suggests that patients with similar demographics and severity of injury vary in their response to traumatic injury. Analysis of the immunological response post-injury showed a sustained pro-inflammatory response with delayed reparative cytokine expression in trauma sensitive patients, while the trauma tolerant patients had an early inflammatory expression with resolution by 72 hours post-injury. Thus, we hypothesize that differential response to non-traumatic injury might serve as a predictive tool for the identification of trauma tolerant and sensitive patients prior to injury. The goal of this research is to test whether immunological changes to inflammatory stimuli can predict tolerance or sensitivity to trauma using an-vitro cell-based assay.

Methods. Splenocytes were isolated from naive C57BL/6 mice and subjected to biological trauma in vitro using LPS $(100 \mathrm{ng} / \mathrm{mL})$ or hypoxic trauma using hydrogen peroxide $(50 \mu \mathrm{M}, 100$ $\mu \mathrm{M}$, and $200 \mu \mathrm{M})$ with or without proinflammatory cytokines, IL-1 $\beta(1 \mathrm{ng} / \mathrm{mL})$, IL-6 $(200 \mathrm{ng} / \mathrm{mL})$, and IL-33 (150 ng/mL). Inflammation and hypoxia were assessed using IL-6 and HIF-1a expression respectively via qPCR 24 hours post-treatment. Cell death and pro-inflammatory cytokine production using multiplex analysis were used to measure outcomes.

Results. Both types of treatments showed increased cell death compared to the control group. qPCR data is pending.

Conclusion. With these studies as a core of the experimental approach, this in vitro cell-based assay will be used to assess immunologic response to inflammatory stimuli across the genetic variation of mouse strains. Findings from this project could enable the development of a clinical test that accurately predicts immunologic response to trauma and related-complications based on patients' sensitivity to pre-traumatic injury. 